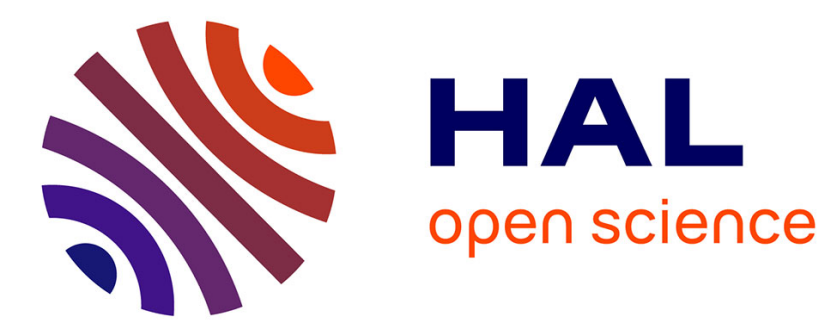

\title{
Eloge du tourisme professionnel : apprendre en voyageant
}

Gilles Brougère

\section{To cite this version:}

Gilles Brougère. Eloge du tourisme professionnel: apprendre en voyageant. Florence Pirard, Sylvie Rayna, Gilles Brougère (dir.). Voyages en petites enfances. Apprendre et Changer, Erès, pp.31-50, 2021, 978-2-7492-6837-8. hal-03585069

\section{HAL Id: hal-03585069 https://hal.science/hal-03585069}

Submitted on 22 Feb 2022

HAL is a multi-disciplinary open access archive for the deposit and dissemination of scientific research documents, whether they are published or not. The documents may come from teaching and research institutions in France or abroad, or from public or private research centers.

Copyright

L'archive ouverte pluridisciplinaire HAL, est destinée au dépôt et à la diffusion de documents scientifiques de niveau recherche, publiés ou non, émanant des établissements d'enseignement et de recherche français ou étrangers, des laboratoires publics ou privés. 


\section{Référence}

Gilles Brougère, «Eloge du tourisme professionnel : apprendre en voyageant » in F. Pirard, S. Rayna, G. Brougère (dir.) Voyages en petites enfances. Apprendre et Changer, Toulouse Erès, 2021, pp. 31-50 


\title{
Éloge du tourisme professionnel : apprendre en voyageant
}

\author{
Gilles BROUGÈRE ${ }^{1}$
}

Ce chapitre a pour objectif d'analyser le voyage d'étude sous le prisme du tourisme. Loin de considérer que ce type de voyage serait antinomique avec lui, il s'agit d'utiliser les recherches menées sur le tourisme pour mieux comprendre les voyages professionnels et leurs effets d'apprentissage. En effet, il ne suffit pas dire rapidement que l'on n'est pas touriste pour ne pas l'être, ni de mépriser le tourisme pour y échapper. D'une part les voyages d'étude, dans les statistiques internationales sous réserve qu'ils intègrent une nuit hors du domicile, sont comptabilisés avec les voyages d'agrément. D'autre part si l'on analyse les logiques sousjacentes on peut voir les grandes similitudes, ce qui permet de considérer à côté du tourisme loisir, le tourisme professionnel qui partage de nombreuses caractéristiques avec le premier. Il ne s'agit pas, loin de là, de rabaisser cette activité, mais sur la base d'une prise en compte des effets d'apprentissage du tourisme que nous avons analysés, de considérer que cela nous permet de mieux comprendre ce qui est en jeu dans un voyage d'étude. Ainsi le court séjour, par opposition au stage ou au séjour long qui permet une immersion, n'a pas de raison d'être perçu négativement; nous nous éloignons d'une conception qui conduirait à penser l'association au tourisme comme impliquant une vision superficielle de la réalité. Nous avons déjà tenté cette approche pour mieux saisir les apprentissages lors des échanges scolaires de courte durée (Brougère, 2018, 2019), nous proposons d'appliquer cela aux voyages d'étude de professionnelles ou d'étudiantes dans le domaine de la petite enfance.

Il existe une longue et ancienne tradition qui fait du voyage une activité formatrice. Comme le signale Boutier (2004) pour Bacon « voyager est pour la jeunesse une forme de l'éducation » et plus loin dans le même texte : «Bacon précise ce qu'il faut faire pour tirer profit d'un voyage, c'est-à-dire pour observer le mieux possible ce qu'il est nécessaire de connaître par l'observation directe » (Boutier, 2004, p. 14). Cette idée positive renvoie à la formation de soi, à la formation par l'expérience, la confrontation à l'autre.

L'exemple toujours évoqué est celui du Grand Tour pour les aristocrates européens :

une expérience partagée qui concerne, à peu d'exceptions près, l'ensemble des aristocraties européennes, lors d'un moment clé de la vie d'un individu, alors que s'achève sa formation et avant qu'il n'entre dans la carrière militaire, administrative ou diplomatique. (Boutier, 2004, p. 8)

L'expression « Grand Tour » est ainsi réservée aux voyages d'éducation. Ce « voyage d'étude » n'exclut pas des moments de divertissement. Mais par sa longueur il a bien des aspects d'un

' Professeur de sciences de l'éducation à l'université Sorbonne Paris Nord 
stage au sein des cours européennes :

Car l'expérience du voyageur ne saurait être réduite à une observation immédiate, facile, du ou des mondes dont le jeune voyageur doit acquérir la connaissance. Pour réussir, elle doit être appliquée à des objets clairement identifiés, puis guidée selon des méthodes adéquates. Elle doit aussi intégrer l'expérience mondaine qui fait elle aussi fortement partie de la culture aristocratique. (Boutier, 2004, p. 13)

Mais Boutier montre également comment progressivement le voyage se fait plus court et plus divertissant, évoluant vers ce qui deviendra, en particulier avec l'essor des villes d'eau, le tourisme.

Le paradoxe est qu'un terme qui a souvent été utilisé de façon péjorative, éloigné de tout apprentissage, évoquant une vision superficielle, moutonnière et sans ouverture à l'altérité, a pour origine quelque chose de sinon opposé, tout au moins différent, lié fortement à la formation de soi (entendu largement comme un soi social et politique), quelque chose d'assez proche du voyage d'étude qui renvoie à un soi professionnel.

Depuis quelques années une nouvelle approche, dans les études sur les pratiques touristiques, met en valeur l'expérience vécue, s'appuie non sur une vision externe, mais essaie de comprendre le point de vue du touriste. J'ai contribué à cela en travaillant sur les apprentissages liés au tourisme dans différents articles et ouvrages (Brougère, 2011, 2012a,b, 2013, 2014a,b, Brougère et Fabbiano, 2014). Je m'appuierai sur ces diverses recherches pour présenter quelques pistes de réflexion. Le point de départ consiste à ne pas faire de différence de nature entre le voyage touristique et le voyage d'étude: les différences internes aux activités touristiques sont plus importantes que celles entre certaines formes de tourisme et les voyages d'étude. Cela permet de mobiliser les résultats de ces recherches pour leur analyse. Un voyage touristique est une forme de voyage d'étude et un voyage d'étude est une forme de tourisme.

La vraie différence se fait entre voyage d'étude et stage (à l'étranger), entre non participation et participation. Le voyageur passe et ne participe pas (c'est celui ou celle qui observe), c'est ce qu'on lui reproche. Les modalités d'apprentissage liées à la participation ne sont donc pas présentes. On peut dans la même logique évoquer la différence entre tourisme et retour au pays (Fabbiano, 2014) ou celle entre un programme de longue durée comme « Voltaire » (Brougère, 2012c) et les échanges scolaires de courte durée (Brougère, 2018); il s'agit du rapport entre participer, être membre, faire avec, et observer à distance, être un hôte pas un membre. Mais il s'agit de montrer que pour autant le voyage court, touristique, centré sur l'observation à distance, n'est pas sans avoir des effets d'apprentissage.

Il convient d'aller au-delà de cette dimension négative car on peut faire fonctionner une autre opposition (et alors le voyage est dans le même camp que la participation à l'activité) entre approche du monde par le moyen de médias, découverte médiatisée (les manuels scolaires, les livres, les films, la télévision, internet, etc.) et celle directe, non médiatisée, qui utilise le corps en déplacement pour la découverte. Le voyage rompt avec la découverte médiatisée, largement 
dominante dans l'éducation (en dehors des stages), en proposant une présence du corps, un corps en mouvement face au monde plutôt qu'un monde en mouvement face au corps statique (télévision et autres médias). On l'aura compris, la question du corps est ici essentielle mais considérer le corps comme vecteur d'apprentissage est en rupture avec la vision cartésienne de la séparation entre corps et esprit et la valorisation de ce dernier dans le processus éducatif (Beckett et Hager, 2002). C'est en cela que la question du voyage est importante car elle ouvre des questionnements essentiels sur l'apprentissage. Pour valoriser les apprentissages d'un voyage d'étude (ou d'un voyage scolaire de courte durée) il faut sans doute penser autrement la question de l'apprentissage.

\section{Quelques éléments de contexte et de méthodologie}

Nous nous sommes intéressés au tourisme, entre autres, pour mettre à l'épreuve des théories de l'apprentissage, en particulier celles relatives à l'apprentissage situé (Lave et Wenger, 1991). Est-ce que les théories utilisées pour comprendre les apprentissages dits informels, par exemple mobilisées pour les crèches parentales (Brougère et Moreau, 2014), sont adaptées aux apprentissages liés au tourisme au-delà du fait d'apprendre les pratiques touristiques ? Il s'agit d'aller au delà du fait que la pratique permet d'apprendre la pratique, que faire des voyages d'étude apprend à faire des voyages d'étude, mais aussi faire des voyages touristiques apprend à faire des voyages d'étude et réciproquement. Il s'agit d'apprentissages pas nécessairement conscients, ni volontaires dans nombre de cas.

Trois terrains ont été menés, l'un concernant le tourisme social en France, le second s'appuyait sur une auto-ethnographie à partir de la rédaction de journaux d'apprentissage en situation touristique. Enfin le dernier, déjà évoqué, concerne des voyages scolaires de courte durée entre France et Allemagne.

Ces recherches s'inscrivent dans l'idée que les situations qui ne sont pas conçues pour apprendre - mais pour un autre objectif lié à la vie quotidienne, au travail ou au loisir dont le tourisme - peuvent permettre d'apprendre, ce que l'on peut appeler apprentissage en situation informelle. En suivant Schugurensky (2007) on peut distinguer des apprentissages tacites (non conscients), des apprentissages fortuits (conscients mais non intentionnels) et de l'autoformation (consciente et intentionnelle en dehors de toute structure éducative formelle).

Les voyages et le tourisme permettent ces différentes situations. On pourrait penser que le tourisme relève plus souvent des deux premières catégories (à l'exception du tourisme culturel qui se rapproche du voyage d'étude) et le voyage d'étude de la dernière (Bouve, 2009). Cependant la situation de voyage, parce que ce n'est pas une situation formelle, génère apprentissages tacites et fortuits et qu'il n'y a pas de solution de continuité entre tourisme et voyage d'étude ou tout au moins, on peut en faire un principe heuristique pour mieux comprendre ce qui se passe. 
On peut, comme pour tout loisir, définir trois modes de relation du voyage en général, ou du tourisme, avec l'éducation :

- Apprendre pour pratiquer le tourisme ou le voyage : apprendre à voyager; on peut alors estimer que la pratique touristique aide au voyage d'étude aussi bien dans son organisation préalable que son déroulement. Si cela renvoie à des situations informelles, on peut également imaginer des cours, un enseignement formel préalable (sur le lieu, le pays, le système préscolaire).

- Apprentissage en situation informelle : on voyage, on visite et on ne cherche pas à apprendre mais on apprend.

- Insérer la pratique (de loisir/ le voyage) dans un projet éducatif, ce qui peut être le cas du voyage d'étude comme du voyage scolaire. Il y a là une tension entre la dimension ordinaire, informelle de l'activité d'une part, et d'autre part un projet éducatif avec des effets d'apprentissage visés.

Nous allons ainsi brièvement présenter certains résultats des recherches menées sur le tourisme puis pour chacun des termes abordés les appliquer aux voyages d'études et à leurs analyses telles qu'on les trouve dans cet ouvrage.

\section{L'observation}

Ce qui caractérise le touriste est une absence de participation à la vie locale, la mise en place d'une distance qui passe par l'observation, comme pour un spectacle, avec un accès limité aux coulisses que les habitants locaux peuvent protéger pour limiter l'impact de la présence des touristes. Le touriste apparaît comme un hôte (au sens de guest), celui qui est reçu sans être membre. Le voyage produit la situation d'hôte, de celui ou celle qui n'est que de passage et auquel on va montrer plus ou moins, tout en essayant de contrôler, voire de limiter ce qu' on lui donne à voir. Il existe un mythe du touriste qui pourrait aller voir les coulisses, mais ce n'est vrai que pour autant qu'on lui donne à voir ceux-ci. Cependant il a accès, comme un local, à tout ce qui se donne à voir dans l'espace public et peut donc dans certaines situations (spectacle, théâtre de rue, carnaval, procession religieuse, etc.) être dans la même posture que la majorité des locaux qui sont spectateurs sans être participants et encore moins organisateurs.

Mais cette distance, que l'on peut parfois critiquer, a un intérêt potentiel : ne pas être impliqué, ne pas avoir à faire et donc être totalement disponible pour regarder (ce qui peut se réduire parfois au coup d'œil), se mettre en situation d'observateur non participant.

C'est bien cette posture que je vis quand je visite des structures d'accueil et d'éducation des jeunes enfants à Reggio Emilia. Or une grande partie de ce qui se fait aussi bien à Reggio qu'à Pistoia se donne à voir à travers la mise en scène et la disposition des objets (telle cette table où sont disposés des objets noirs), le choix du mobilier, l'aménagement esthétique (des voiles séparent des espaces), la mise en valeur des travaux d'enfants accrochés comme des œuvres d'art, la documentation ou tout au moins la façon dont elle peut ici et là se donner à voir, les 
lumières projetées, éléments visibles du travail des enfants avec différentes sources lumineuses ou encore le rangement des objets dans les différents ateliers. Le regard et l'observation sont ici une entrée dans la pédagogie des lieux que ne peuvent tout à fait permettre des textes voire des photographies ou des films. Déambuler et regarder est un premier moment essentiel pour accéder au sens de ces lieux et de leurs pratiques. À cela s'ajoute l'observation des actions des enfants comme des professionnelles.

C'est en cela que le voyage d'étude est du tourisme, par ce passage rapide, ce primat de l'observation qui le distingue du stage, de l'investissement par participation. Ce qui valide une telle démarche c'est qu'il n'est pas possible de s'inscrire partout comme participant, faute de temps, de moyens mais aussi d'acceptation de celui ou celle qui accueille (host) qui veut bien un observateur mais pas nécessairement une personne qui vient agir dans son monde.

Le visiteur va gérer d'abord son regard, alterner des coups d'œil et des regards plus focalisés et durables, mais ce regard, contrairement à celui qui renvoie aux médias, est porté par un corps qui déambule, qui va découvrir des éléments que d'autres considéreraient comme sans intérêt. Et le fait qu'il ne comprenne pas toujours la langue utilisée par les acteurs renforce l'importance du regard et en conséquence du visible.

Mais si le regard est essentiel, l'observation implique tous les sens : " On est plongé dans une expérience visuelle, sonore, olfactive. Tout notre corps, nos sens y participent : c'est un engagement de toute notre personne. »(Airoldi, Sanou et Fracheboud, dans cet ouvrage). L'exploration proposée ne se limite pas à celle du regard pour autant que l'on puisse se mouvoir dans les lieux : « nos hôtes nous ont permis de déambuler, toucher, explorer. Tous nos sens ont été en éveil, nous avons pu appréhender la vie des lieux, la sentir » (Andrys et Rharbi dans cet ouvrage)

\section{L’émerveillement}

Le travail sur le tourisme social a révélé l'importance de l'émerveillement. Etre étonné, émerveillé, découvrir quelque chose de différent est un élément essentiel qui peut rendre possible l'apprentissage. J'ai pu le vérifier dans mon propre journal à travers par exemple Ravenne et les mosaïques (Brougère, 2014b). Cet émerveillement est lié en partie à la relation directe sans médiation et ne doit pas être confondu avec l'authenticité. Ce qui émerveille peut être une fabrication, un spectacle, un parc d'attraction. Or des éducatrices de jeunes enfants en voyage à Pistoia évoquent l'émerveillement qui les saisit lors de visites.

Cet émerveillement renvoie à la curiosité et n'est pas sans être en relation avec les cabinets de curiosité dont le but était de produire, à partir d'éléments recueillis tout autour du monde, l'étonnement et l'émerveillement devant les merveilles de la nature ${ }^{2}$. Forme ancienne de la

\footnotetext{
${ }^{2}$ Sur cette question on peut se reporter aux analyses proposées par le catalogue du Musée des confluences (2014) sous le beau titre Dans la chambre des merveilles.
} 
construction du savoir, préscientifique pourrait-on dire, mais qui fonctionne aujourd'hui encore en dehors du monde de la science. D'autant plus que ce que propose Pistoia dans ses agencements d'objets renvoie également aux cabinets de curiosité cette fois avec l'idée d'utiliser l'étonnement, l'émotion, l'émerveillement en direction des enfants pour rendre possible des apprentissages. À la découverte de l'inattendu, l'émotion peut être déclencheur d'apprentissage et de changement.

Les comptes rendus des voyages d'étude évoquent à plusieurs reprises cet émerveillement lié à d'autres émotions proches, clefs de l'apprentissage :

Les échanges avec les autres participantes du voyage sont riches et les impressions sont semblables. Les émotions mobilisées appartiennent au même registre, il s'agit d'émerveillement, d'enthousiasme, de motivation et nous verrons que celles-ci seront le moteur de réflexions, d'apprentissages et de changements [...] Nous prononcerons (et entendrons prononcer) le mot «émerveillement» à de multiples reprises, et c'est bien ce type d'émotion qui prédomine (Guinchard Hayward et Desponds Theurillat dans cet ouvrage)

Cet émerveillement est en relation avec l'importance du regard et de l'observation évoquée dans la section précédente et renvoie à une symétrisation entre les apprentissages des enfants et ceux des visiteurs :

Travailler avec les enfants, c'est avoir peu de certitudes et de nombreuses incertitudes; ce qui nous sauve, c'est la recherche, le refus de perdre le langage de l'émerveillement qui persiste au contraire dans les yeux et l'esprit des enfants. [propos attribués à Malaguzzi par Alfredo Hoyuelos (2004, p.6)]

\section{Le guidage}

Le tourisme est une pratique guidée : livres, guides professionnels ou amateurs, guidage plus informels des hôtes, ceux qui reçoivent les touristes, des passants, des autres touristes qui peuvent conseiller d'aller à tel ou tel endroit.

Le guidage renvoie à plusieurs caractéristiques du tourisme qui sont sans doute au-delà partagées avec d'autres voyages. Le touriste dispose de temps libre : que peut-on faire quand il n'y a pas une contrainte qui définisse ce qu'il convient de faire, d'où l'importance d'une orientation. Il se trouve dans un espace inconnu et a besoin de repères pour savoir où aller et comment : les moyens de transport sont un élément essentiel de la pratique touristique.

Le voyage d'étude est également guidé et c'est essentiel. Ce qui est guidé c'est entre autres la visite, la pratique même du voyage et non pas l'apprentissage, mais c'est ce qui peut rendre possible l'apprentissage.

La visite « intelligente » est toujours guidée (dans une acception très large de ce terme et surtout non morale). Il faut être guidé pour observer, que des éléments puissent être extraits, traduits du visible. [...] Ces guides multiples, humains comme non humains, matériels ou idéels, permettent 
d'instruire un rapport plus intentionnel à l'espace, de réduire la complexité phénoménale de la découverte. (Bossé, 2015 p.97)

Il faut sans doute dans le voyage d'étude comme dans le tourisme distinguer les accompagnateurs (plus proche des visiteurs, des guests) des guides (du côté des hosts), même si la frontière est floue et instable car l'accompagnateur peut connaître les lieux, y avoir déjà été guidé et ne découvre pas toujours ce que ceux qu'il accompagne découvrent. Les effets d'apprentissage ne se limitent pas aux visiteurs, accompagnateurs compris, mais également concernent les guides. En effet adopter la posture de guide c'est aussi être visiteur et l'on peut voir ce que l'on n'avait pas vu : le guide apprend en guidant. De plus il est valorisant de recevoir des visiteurs (comme l'est un site touristique valorisé par ce simple fait). Toutes les villes, ou presque, veulent aujourd'hui être touristiques, une façon de mettre en évidence leurs atouts et spécificités, culturelles ou autres. On peut considérer qu'il en est de même pour une structure d'accueil pour laquelle la présence de visiteurs peut être vue comme un gage de qualité. On peut souligner la relation forte à Reggio Emilia entre la qualité attribuée au système préscolaire municipal et la présente de visiteurs dont le voyage est organisé et guidé selon un modèle qui renvoie en partie à l'accueil de touristes.

Le guide peut avoir comme fonction de protéger les visités de comportements inappropriés ou non voulus de la part des visiteurs. Les participants à un voyage d'étude peuvent être perçus comme envahissants selon les mêmes critiques qui concernent les touristes en général. On sent la tension entre l'intérêt de recevoir et la peur d'être envahi qui conduit à organiser les circuits, les visites et donc les éloigner de ce fait de toute participation, mettant en évidence la différence entre ceux qui visitent et ceux qui reçoivent.

Le tourisme comme le voyage d'étude est marqué par ce qu'on peut appeler, à la fois par opposition et par relation à la participation guidée (Rogoff, 1999; Brougère, 2009), l'exploration guidée qui va permettre d'orienter le regard et de rendre possibles certains apprentissages.

\section{Apprendre par corps, le rôle central du corps dans l'apprentissage}

« La première ressource du visiteur est son propre corps, la référence dont il dispose au monde sensible, son tout premier instrument de mobilisation de ce monde » (Bossé, 2015, p. 65). Le rôle du corps est essentiel. Au-delà du regard c'est une présence du corps et de tous les sens qui renvoient (rôle des bruits et des odeurs par exemple) à un corps qui se déplace, qui utilise différents modes de transport, marqué par le principe du sight seing, qui peut connaître la fatigue, va rechercher des moments et des supports pour se reposer. On apprend par le corps mais aussi du corps. Ainsi ai-je évoqué l'exemple de la découverte, sur le site archéologique de Mycènes, de la porte aux lions, d'une construction qui ne prend sens que par rapport au corps. Le fait d'avoir vu des images ne donne pas une vision précise du lieu et de ses bâtiments, alors que de le traverser, d'en prendre la dimension avec son propre corps permet une nouvelle connaissance plus sensible du site. 
L'observation se fait de visu, in corpore, en étant présent ce qui distingue d'une vision issue de média (quel que soit ce média). Et il semble qu'il y ait là des effets perçus dans le tourisme et qui s'appliquent très bien au voyage d'étude. Le tourisme permet ce que ne peuvent offrir des livres. Ce n'est pas la même chose de visiter les structures de Reggio Emilia, Pistoia ou de Gand et de lire un livre. Il y a une expérience corporellement enracinée, multi-sensorielle, qui offre une découverte et un apprentissage différents : « La visite est pour le visiteur une épreuve spatiale liée à sa présence in situ. Il s'agit donc de réfléchir la visite comme une expérience avant tout corporelle et sensible, comme une mise à l'épreuve du corps-visiteur » (Bossé, 2015, p. 53) et plus loin dans le même ouvrage : «L'engagement corporel dans l'espace se traduit en effet avant tout par des impression kinesthésique et des émotions » (p. 57).

Cet engagement au-delà du visible, permet de s'imprégner d'une atmosphère, des odeurs,... et garantit un engagement d'autant plus important qu'il implique le corps dont il ne peut jamais être déconnecté. Réciproquement la visite implique d'offrir au corps des possibilités de circulation, de mouvement. Ainsi peut-on agir, expérimenter avec son corps :

[...] peu d'entre nous ont d'ailleurs résisté au plaisir de se saisir de la balle, de faire glisser une voiture le long d'une rampe, ou de communiquer avec une collègue à l'aide du tubo telefonante qui invite à s'en saisir...! Nous aussi devenions des apprenants actifs dans ces environnements ! (Guinchard Hayward et Desponds Theurillat dans cet ouvrage)

L'expérience conduit ainsi à « apprendre à partir de nos corps, en nous déplaçant, en ressentant, en nous émerveillant. » (Andrys et Rharbi dans cet ouvrage). Et Vincent Enrico (dans cet ouvrage) fait remarquer combien le corps est sollicité par la situation, devient un corps fatigué, « mis à l'épreuve de façon inhabituelle " comme nous l'avions déjà remarqué lors des séjours scolaires (Brougère, 2018).

Le voyage prend la forme d'un circuit, et implique l'organisation des circulations car s'il s'agit d'aller d'un lieu à une autre, il importe également que ces corps en mouvement ne perturbent pas l'activité que l'on vient observer. Cela peut impliquer une adaptation et donc une modification des activités elles-mêmes ou des logiques de régulation pour limiter cette présence de corps d'autant plus que dans les structures d'accueil de la petite enfance s'ajoute la présence de corps adultes dans un univers conçu surtout pour des corps d'enfants. Ce corps qui permet l'accès peut se faire encombrant et il y a des lieux où les corps des visiteurs n'ont pas accès. Les accueillants peuvent édicter des règles limitant l'accès de certains lieux qui ne sont pas ouverts à la visite, mais des espaces peuvent être conçus pour ne rendre possible l'accès qu'à des corps d'enfants.

\section{La photographie}

La photographie est fortement liée au corps et a souvent pour rôle, renforcé par la possibilité d'afficher aujourd'hui les photos en temps réel, de montrer sa présence dans le lieu. Entre 
documentation et performance, la photographie n'est pas seulement une trace, une mémoire mais fait partie de l'action en situation. On trouve bien ces deux aspects dans le voyage d'études, la photographie témoignant d'une performance collective, être ensemble dans tel lieu, et permettant de documenter le voyage.

Le voyage d'étude laisse des traces qui ont pour but de garder la mémoire d'un moment collectif, d'une présence dans un lieu précis, de rappeler que «j'y étais » tout autant que de documenter ce que l'on a vu, aider la mémoire et pouvoir expliquer à ceux qui n'étaient pas là ce que l'on a retenu, éventuellement entrer dans un dynamique d'appui à la transformation de sa pratique, de son institution.

Mais elle en montre tout autant les limites, les traces ne pouvant remplacer la confrontation avec une réalité. On retrouve la logique médiatique que le voyage cherche à rompre. Ne pas avoir été du voyage c'est ne pas avoir partagé cette expérience, et on ne peut s'étonner du décalage tout autant avec le moment collectif de partage face à un ailleurs qu'avec la réalité que l'on ne découvre que de façon médiate.

\section{La comparaison}

La tendance du touriste à comparer avec ce qu'il connait, chez lui ou à l'occasion de voyages antérieurs, est ce dont on se moque souvent, mais qui est sans doute un processus essentiel d'apprentissage en situation :

Tout au long du voyage nous n'avons pas pu nous empêcher de faire des comparaisons avec ce que nous connaissions en France, et nous nous sommes demandés qu'elle aurait été notre réaction face à un groupe d'étrangers qui se serait présenté dans notre structure. (Ambrus et al. dans cet ouvrage)

Ainsi le voyage d'étude permet de comparer avec les structures où l'on travaille mais également les structures entre elles, le voyageur pouvant être plus sensible à ce qui les rassemble, les acteurs locaux à ce qui les sépare. La comparaison structure une partie des questions que l'on peut adresser aux acteurs locaux et des conversations que l'on a aussi bien avec les acteurs locaux qu'avec les autres visiteurs. Importe en conséquence la différence ou la distance avec son expérience quotidienne :

Choisir de «partir loin » n'est pas considéré comme indispensable. Le choix de la destination est moins une question d'éloignement géographique que celle d'une distance entre des pratiques mises en œuvre par les voyageuses dans leur lieu d'accueil et celles qu'elles découvrent dans le lieu visité. (Pirard et al., dans cet ouvrage)

C'est bien la possibilité de comparer et de mettre en évidence des différences qui structure au moins une partie des voyages d'étude et leurs effets d'apprentissage. 


\section{Assumer la dimension touristique}

Nous avons évoqué la proximité du voyage d'étude avec le voyage scolaire (Brougère, 2018, 2019). Dans ce cas, le terme de tourisme est refusé par les responsables et acteurs éducatifs, mais si l'on regarde les programmes on s'aperçoit que les lieux visités (à Paris, à Berlin ou à Lyon) sont ceux visités par les touristes. Certes le guidage peut être en partie spécifique (le livre où les élèves ont appris le français pour les Allemands à Paris, ou le projet autour de l'assassinat des Juifs à Berlin), mais c'est bien observer sans participer (sinon à la marge dans la vie de famille mais pour peu de temps), participer aux rituels des touristes, prendre des photos. On voit le rôle du corps mais aussi l'importance du groupe, de visiter ensemble. En revanche, pour les jeunes il n'y a pas de conflit, le tourisme est plutôt valorisé et pas antinomique du fait d'apprendre, apprendre autrement en déambulant.

On peut donc considérer que le voyage d'étude est aussi un voyage touristique (et pas seulement pour les statistiques officielles) et ceci d'une part parce que les visites professionnelles peuvent être considérées comme des visites touristiques à finalité spécifique, d'autre part parce qu'autour des visites professionnelles peuvent se greffer des visites proches du tourisme ordinaire, comme la visite guidée de Lyon (Lefebvre et Legros, dans cet ouvrage). Quand je visite des écoles à Bristol, je visite Bristol en même temps. Il n'y a pas rupture entre les deux dimensions. On peut ainsi mettre en évidence les différences dimensions du voyage : visites touristiques et visites professionnelles.

Ainsi il n'est pas possible de visiter les structures de Pistoia sans découvrir dans le même temps une ville italienne au riche patrimoine, mais surtout accueillante pour les enfants :

Cette importance de l'enfance dans la Cité nous est aussi apparue lors de nos pérégrinations de touristes à travers la ville, par petites touches, comme si cela allait de soi que les enfants ont droit à une place, une place active, que ce soit sur la Piazza del Duomo, au Musée, au café ou à la librairie. Cette prise en compte des enfants, alliée à l'idée que les structures qui leur sont dédiées ainsi qu'à leur famille ont un rôle à jouer, semble ici une évidence, de même que la visibilité des enfants dans la ville. (Guinchard Hayward et Desponds Theurillat dans cet ouvrage)

Cette dimension est également jugée essentielle par les responsables des visites à Pistoia, dans la mesure où les structures d'accueil de la petite enfance sont totalement liées à la ville :

Les programmes de visites incluent des lieux artistiques, culturels et historiques, pour montrer les liens existant entre les structures éducatives de la petite enfance et la ville. Comme l'indique notre Charte des structures éducatives, toute la ville est impliquée dans la tâche éducative, elle représente un macro-contexte éducatif. Découvrir ses caractéristiques ouvre des chemins de connaissances pour une meilleure lecture du contexte culturel, historique et artistique dans lequel les structures de la petite enfance s'inscrivent. (Cappellini, Giovannini et Contini, dans cet ouvrage)

La visite touristique contribue ainsi à mieux saisir le contexte et enrichit la visite professionnelle. Il n'y pas opposition mais complémentarité entre les deux types de visite. C'est 
le même statut de visiteur qui se déploie dans l'ensemble des espaces, professionnels ou non. Cela conduit sans doute à une unité du regard, dans la traversée des divers lieux visités, quelle qu'en soit la raison, professionnelle, touristique ou simplement technique dans la nécessité de se déplacer en deux structures. C'est aussi une occasion de promouvoir la ville, d'en faire découvrir les richesses qui peuvent générer un tourisme de loisir dans cette ville toscane.

Les dimensions évoquées dans les différentes sections ci-dessus sont présentes dans les deux types de visites qui, de plus, peuvent ne pas être totalement séparés, certaines structures pouvant se trouver dans un quartier doté de valeurs touristiques traditionnelles liées au patrimoine.

Que la finalité soit touristique, au sens de loisir, ou professionnel, il y a relation à l'altérité, la différence, même si l'encadrement des pratiques peut avoir pour objectif de limiter l'effet en évitant une plongée trop brutale dans cette altérité. La sélection des lieux, le guidage ont tout autant pour fonction de faire découvrir que de rendre possible la découverte de quelque chose de différent. Reste que dans les deux cas l'effet peut-être de questionner son propre monde : «L'expérience de l'ailleurs se caractérise par le vécu de situations dont la propriété est de déroger aux habitudes, et d'interroger les évidences qui produisent le familier » (Breton, 2017, p. 28)

\section{Les apprentissages de ceux qui reçoivent}

Autre point commun avec le tourisme, déjà évoqué à l'occasion du guidage, est l'idée que les apprentissages ne sont pas réservés aux visiteurs, mais concernent également les visités même si cette question est encore peu présente dans la littérature. Il est plus souvent question de la déstabilisation voire de la destruction des milieux visités par le tourisme que d'apprentissages, les plus évidents étant les apprentissages linguistiques. Et pourtant, qu'il s'agisse de tourisme loisir ou professionnel, cela suppose une mise en scène, un travail de préparation à la visite : « on oscille entre pédagogie, valorisation et marketing (le visiteur comme client). La visite comme outil de performation » (Bossé, 2015, p. 24). Faire visiter demande un travail de la part de celui qui reçoit.

Guider implique de regarder autrement mais aussi développe une réflexivité sur ce que l'on fait visiter qui devient, de ce fait, plus intéressant : «adopter la posture de guide c'est aussi être visiteur et l'on peut voir ce que l'on n'avait pas vu » (Bossé, 2015, p. 69)

Les visiteurs évoquent la qualité de l'accueil qui relève d'une volonté de bien recevoir : « La qualité de l'accueil tient à cette capacité à rencontrer l'autre. Dès les premiers contacts, l'approche pédagogique de Pistoia est incarnée par tous les protagonistes, parents, professionnels, et enfants. » (Airoldi, Sanou et Fracheboud, dans cet ouvrage). On peut alors se demander si l'accueil d'étrangers ne fait pas partie du curriculum des structures au sens où accueillir permet d'une part d'apprendre l'accueil (ce qui est central dans un lieu d'accueil), d'autre part de motiver des activités liées à l'accueil (et ceci aussi bien pour les enfants que pour les adultes). 
C'est ce que souligne l'exemple de Pistoia :

Les équipes ont développé depuis longtemps une tradition de pratiques d'accueil hospitalières : comment se préparer, présenter les structures, les enfants, les enseignantes, gérer le temps de visite des groupes, savoir que faire et qu'offrir. Elles savent l'importance d'une réflexion permanente sur les modalités d'accueil [...] Un accueil empathique amène, en effet, non seulement une approche agréable des lieux mais aussi une familiarité, une intimité, propice au dialogue et à la réciprocité. (Cappellini, Giovannini et Contini, dans cet ouvrage)

Cet accueil qui implique également les enfants suppose un contact avec des personnes dont les opinions diffèrent et peut être vécue comme une auto-formation :

C'est comme si chacune de nous, chaque fois qu'on accueille des visiteurs, on se trouvait dans une auto-formation, qu'on partage ensuite avec son équipe.

Nous ne sommes pas immédiatement conscientes des acquis professionnels qui découlent de l'accueil des visiteurs.

C'est un parcours d'hospitalité qui nous amène à nous développer continuellement en tant que professionnelles et en tant que personnes (paroles d'enseignantes de Pistoia, Cappellini, Giovannini et Contini, dans cet ouvrage)

A travers ces différents apports le voyage professionnel permet également de poser de nouvelles questions et un nouveau regard sur la question de l'accueil dans les formes traditonnelles du tourisme entendu comme loisir.

\section{Education informelle et formalisation}

Nous avons mis l'accent sur la dimension informelle des apprentissages, mais les voyages d'étude renvoient à une formalisation potentielle des apprentissages, au moins dans certains cas. On peut, dans un premier temps, évoquer la notion paradoxale d'éducation informelle : elle renvoie à la présence d'objectifs pédagogiques mais en utilisant les moyens de l'apprentissage en situation informelle, en l'absence de toute mise en place de dispositifs éducatifs, comme Schugurensky (2009) l'évoque à propos budget participatif de Porto Alegre. Dans notre cas, cela signifierait que l'on fait confiance au voyage, aux visites sans formaliser les apprentissages visés. Il s'agit de s'appuyer sur l'effet visite et aux échanges que cela peut susciter au sein du groupe.

Il peut exister des tentatives de cadrer pédagogiquement le séjour, mais le voyage ne peut être totalement contrôlé, il implique des imprévus et un éloignement de l'institution que certains peuvent regretter. Ceux qui voyagent échappent d'une certaine façon à l'institution, ne sont pas totalement contrôlables. La formalisation hors les murs rencontre des limites. Reste que la volonté de justifier le voyage (ce qui suppose qu'il ne pourrait se justifier de lui-même), d'éviter les critiques qui renvoient au tourisme et aux vacances conduit souvent à formaliser, préparer, encadrer, pédagogiser. 
Les voyages d'étude connaissent ainsi une tension entre leur dimension informelle qui renvoie à la logique touristique et la volonté d'en faire des dispositifs éducatifs que l'on pourrait justifier aux yeux de ceux pour qui l'apprentissage ne peut que résulter de dispositifs formels.

Si l'analyse met en évidence les logiques d'apprentissage en situation informelle, et ceci à plusieurs niveaux et pas seulement en relation avec le lieu visité mais également au niveau de la dynamique du groupe que nous n'avons pas évoquée, elle montre tout autant une volonté de cadrer, de formaliser et témoigne d'une certaine peur de l'informel. Comme l'évoquent dans cet ouvrage Florence Pirard et al. :

[...] l'analyse fait ressortir le caractère singulier de l'expérience pour les participants. Il faut noter que ceux-ci ne concentrent pas nécessairement toute leur attention sur l'objet initialement prévu par les accompagnateurs et se laissent interpeller par d'autres préoccupations en rapport avec leur vécu et l'amélioration d'une qualité d'accueil comme par exemple l'investissement de l'espace extérieur.

Le voyage peut être certes formalisé et devenir un dispositif éducatif, mais son caractère total, holistique (Beckett et Hager, 2002), qui consiste à plonger un corps dans une réalité complexe fait qu'il est toujours dans une logique d'apprentissage en situation informelle selon des modalités qui sont celles mises en évidence dans le tourisme. Il y a sans doute des apprentissages non conscients ou tacites qui ne deviendront explicites qu'ultérieurement, traces de cette aventure qui ne sont pas visibles dans l'immédiat, des apprentissages fortuits qui sont liés à tout ce que peut avoir d'imprévisible un voyage, des visites et enfin une logique d'autoformation au sens où chacun dirige son regard, déambule, pose des questions en fonction des questions qui sont les siennes.

\section{Conclusion}

« Si nous faisons du tourisme, c'est pour aller interroger un certain nombre de lieux dont nous savons qu'ils ont quelque chose à nous dire » (Butor, 2014 [1993], p. 136) Cette vision du tourisme par Butor dont il faut noter le côté positif à une époque où la critique, en particulier chez les intellectuels, est de mise, s'applique on ne peut mieux aux voyages d'étude.

Il faut admettre qu'il s'agit d'une forme de tourisme que nous pouvons appeler " professionnel » au sens où il renvoie à une logique liée à l'activité professionnelle, marquée par la découverte et l'exploration guidée comme nombre de formes du tourisme. Les effets d'apprentissage sont sans doute liés à cette exploration guidée mais qui reste ouverte à la déambulation, au hasard, car guider ce n'est pas encadrer, c'est tout au plus montrer une direction, orienter, mais il est toujours possible de regarder ailleurs, de prendre un autre chemin.

Les apprentissages sont d'une grande diversité, en relation avec la multiplicité des activités concrètes qui sont mobilisées à l'occasion d'un voyage, d'une visite. Nous en avons suggérés 
quelques uns. On peut ajouter comme exemple de cette variété l'organisation comme facteur d'apprentissage ou «apprendre en organisant» (Ambrus et al., dans cet ouvrage) que l'on découvre également chez les touristes qui auto-organisent leurs voyages.

Pour finir nous pourrions évoquer à nouveau la symétrie entre apprentissage dans le préscolaire et voyage d'étude en considérant que l'exploration guidée est une dimension essentielle de la pédagogie préscolaire, en particulier à Pistoia et Reggio Emilia. On peut donc concevoir l'éducation préscolaire comme un voyage de découverte et le voyage d'étude comme une façon de partager avec les enfants une pédagogie qui accorde une place importante aux situations informelles du point de vue éducatif, qui accepte la rencontre, la visite et l'improvisation, qui sait donner une place au corps et à tous les sens. Cela renvoie à une vision holistique de l'éducation que l'on peut retrouver à différents niveaux (Beckett et Hager, 2002).

\section{Références bibliographiques}

BECKETT, D.; HAGER, P. 2002. Life, Work and Learning. Practice in postmodernity, Londres, Routledge.

BOSSE, A. 2015. La visite. Une expérience spatiale, Rennes, PUR

BOUVE, C. 2009. «Le voyage d'étude comme pratique autoformative des professionnels de la petite enfance », dans S. Rayna, C. Bouve, P. Moisset (sous la direction de) Pour un accueil de qualité de la petite enfance : quel curriculum ?, Toulouse, Erès.

BOUTIER, J. 2004. " Le grand tour : une pratique d'éducation des noblesses européennes (XVI ${ }^{\mathrm{e}}-\mathrm{XVIII}{ }^{\mathrm{e}}$ siècles). Le voyage à l'époque moderne », Cahiers de l'Association des Historiens modernistes des Universités, ${ }^{\circ} 27$, pp. 7-21.

BRETON, H. 2017. « Se former par l'expérience de l'ailleurs : situations d'indétermination et acquis du voyage ». Education permanente, n`211, pp. 27-37

BROUGÈRE, G. 2009. «Une théorie de l'apprentissage adaptée : l'apprentissage comme participation », dans G. Brougère, Anne-Lise Ulmann (sous la direction de) Apprendre de la vie quotidienne, Paris, PUF, pp. 267-278.

BROUGÈRE, G. 2011. Qu'apprennent des personnes aidées en vacances ? Communication au congrès de l'AREF, Genève, septembre 2010, publié dans les actes, $<$ https://plone2.unige.ch/aref2010/symposiums-longs/coordinateurs-en-b/tourisme-etapprentissages/Quapprennent $\% 20 \mathrm{des} \% 20$ personnes $\% 20$ aidees $\% 20 \mathrm{en} \% 20$ vacances.pdf $/$ vie $\underline{\mathrm{w}}>$

BROUGÈRE, G. 2012a. S'observer comme touriste apprenant, Communication au colloque «Tourisme et apprentissages », Université Paris 13, avril 2011, publié avec les actes, $<\underline{\text { http://www.univ-paris13.fr/experice/brougere/Brougère }>}$

BROUGÈRE, G. 2012b. « Pratiques touristiques et apprentissages », Mondes du tourisme, n5, pp. 62-75.

BROUGÈRE, G. 2012c. «Participation, learning and intercultural experience », dans C. Baraldi, V. Ieverse (sous la direction de) Participation, Facilitation and Mediation. Children and Young People in Their Social Contexts, Londres, Routledge, pp.180-196 
BROUGÈRE, G. 2013. « Learning the practice, learning from the practice: tourist practices and lifelong education », International Journal of Lifelong Education, Vol. 32, n²1, pp. 93-106

BROUGÈRE, G. 2014a. "Les apprentissages guidés du touriste », dans G. Brougère, G. Fabbiano, Apprentissages en situation touristique, Lille, Presses Universitaire du Septentrion, pp. 87-97

BROUGÈRE, G. 2014b. « Soi-même comme touriste apprenant. Essai d'auto-ethnographie », dans G. Brougère, G. Fabbiano (sous la direction de) Apprentissages en situation touristique, Lille, Presses Universitaire du Septentrion, pp. 155-180.

BROUGÈRE, G. 2018. «Corps et lieux dans les échanges ou l'échange scolaire comme expérience touristique », dans G. Brougère, $\mathrm{C}$. Wulf (sous la direction de) A la rencontre de l'autre. Lieux, corps, sens dans les échanges scolaires, Paris, Téraèdre, pp. 137-194.

BROUGÈRE, G. 2019. « Les échanges scolaires sont-ils solubles dans le tourisme ou apprendre en faisant le touriste », dans E. Peyvel, L'éducation au voyage. Pratiques touristiques et circulations des savoirs, Rennes, PUR.

BROUGÈRE, G., FABBIANO, G. 2014. «Introduction. Apprendre en situation touristique, défis et enjeux », dans G. Brougère, G. Fabbiano (sous la direction de) Apprentissages en situation touristique, Lille, Presses Universitaire du Septentrion, pp. 9-29.

BROUGÈRE, G. et MOREAU, A. 2014. «Participation parentale, pratiques partagées et diversité », dans S. Rayna, G. Brougère (sous la direction de) Petites enfances, migrations et diversités, Bruxelles, P.I.E. Peter Lang, pp. 137-163.

BROUGÈRE, G.; ULMANN A.-L. (sous la direction de) 2009. Apprendre de la vie quotidienne, Paris, PUF.

BUTOR, M. (2014 [1993]). Improvisations sur Michel Butor. L'Écriture en transformation, Paris, Editions de la Différence.

FABBIANO, G. (2014) «Être là. Les voyages au pays d'origine des familles harkies entre expérience mémorielle et situations d'apprentissage », dans G. Brougère, G. Fabbiano (sous la direction de) Apprentissages en situation touristique, Lille, Presses Universitaire du Septentrion, pp. 143-154.

HOYUELOS, A. 2004, «Une transgression esthétique », Enfants d'Europe, nº, pp. 6-7.

LAVE J. et WENGER E. 1991, Situated Learning. Legitimate Peripherical Participation, Cambridge University Press.

MUSEE DES CONFLUENCES 2014. Dans la chambre des merveilles, Paris, Flammarion

ROGOFF B. 1999. Apprenticeship in thinking: Cognitive development in social context, Oxforf, Oxford University Press.

SCHUGURENSKY, D. 2007. "Vingt mille lieues sous les mers : les quatre défis de l'apprentissage informel », Revue française de pédagogie, $\mathrm{n}^{\circ} 160$, pp. 13-27

SCHUGURENSKY, D. 2009. «Apprendre en faisant : démocratie participative et éducation à la citoyenneté », dans G. Brougère, A.-L. Ulmann (sous la direction de) Apprendre de la vie quotidienne, Paris, PUF, pp. 207-219. 